Virginia Commonwealth University VCU Scholars Compass

2004

\title{
Appearance of bulk properties in small tungsten oxide clusters
}

Q.Sun

Virginia Commonwealth University

B. K. Rao

Virginia Commonwealth University

P. Jena

Virginia Commonwealth University, pjena@vcu.edu

See next page for additional authors

Follow this and additional works at: http://scholarscompass.vcu.edu/phys_pubs

Part of the Physics Commons

Sun, Q. Rao, B. K., Jena, P., et al. Appearance of bulk properties in small tungsten oxide clusters. The Journal of Chemical Physics 121, 9417 (2004). Copyright @ 2004 AIP Publishing LLC.

\section{Downloaded from}

http://scholarscompass.vcu.edu/phys_pubs/176

This Article is brought to you for free and open access by the Dept. of Physics at VCU Scholars Compass. It has been accepted for inclusion in Physics Publications by an authorized administrator of VCU Scholars Compass. For more information, please contact libcompass@vcu.edu. 
Authors

Q. Sun, B. K. Rao, P. Jena, D. Stolcic, Y. D. Kim, Gerd Gantefor, and A. W. Castleman Jr. 


\title{
Appearance of bulk properties in small tungsten oxide clusters
}

\author{
Q. Sun, B. K. Rao, ${ }^{\text {a) }}$ and P. Jena \\ Department of Physics, Virginia Commonwealth University, Richmond, Virginia 23284-2000 \\ D. Stolcic, Y. D. Kim, ${ }^{\text {b) }}$ and Gerd Gantefor \\ Department of Physics, University of Konstanz, D-78457 Konstanz, Germany
}

A. W. Castleman, Jr.

Departments of Chemistry and Physics, Pennsylvania State University, College Station, Pennsylvania 16802

(Received 2 July 2004; accepted 25 August 2004)

\begin{abstract}
Contrary to the conventional understanding that atomic clusters usually differ in properties and structure from the bulk constituents of which they are comprised, we show that even a dimer of tungsten oxide $\left(\mathrm{WO}_{3}\right)_{2}$ possesses bulklike features and the geometry of a small cluster containing only 4 tungsten and 12 oxygen atoms bears the hallmarks of crystalline tungsten oxide, $\mathrm{WO}_{3}$. This observation, based on a synergistic approach involving mass distributions under quasisteady state conditions, photoelectron spectroscopy, and first principles molecular orbital theory, not only illustrates the existence of a class of strongly covalent or ionic materials whose embryonic forms are tiny clusters but also lends the possibility that a fundamental understanding of complex processes such as catalytic reactions on surfaces may be achieved on an atomic scale with clusters as model systems. () 2004 American Institute of Physics. [DOI: 10.1063/1.1807374]
\end{abstract}

\section{INTRODUCTION}

As atoms are successively removed from materials, a point is reached when the properties of the remaining particle no longer resemble those of its bulk. ${ }^{1-6}$ The length scale when this occurs is typically a few nanometers. Atomic clusters belong to this class of materials. It is well known that the geometries and electronic properties of clusters are very different from their bulk. For example, clusters of metallic elements exhibit fivefold symmetry and icosahedric growth, ${ }^{7}$ which is nonexistent in crystals. The building block of silicon clusters is a tricapped trigonal prism (TTP), ${ }^{8,9}$ which is completely different from the bulk diamond structure. Some of the most stable carbon clusters have the famous fullerene geometry ${ }^{1}$ while bulk carbon exists in either planar or tetrahedral structures.

These interesting phenomena in nanodimensions raise a fundamental question: What is the critical cluster size at which electronic and geometric structures converge to the bulk properties? Previously, experimental and theoretical studies have been performed to provide an answer to this question. For coinage metals such as $\mathrm{Au}, \mathrm{Ag}$, and $\mathrm{Cu}$, it has been observed that the clusters consisting of less than 50-60 atoms exhibit dissimilar electronic structures compared to the respective bulk counterparts, and only for larger clusters, bulklike electronic structures appear. ${ }^{10}$ In full shell systems the interaction between the neighboring groups in bulk is relatively weak, resulting in fast convergence to bulk properties. Among others, systems to be named are noble gases, alkali halides, water, etc. In contrast to the pure metal clusters and full shell systems, it is unclear so far, that how fast

\footnotetext{
${ }^{\text {a) }}$ Deceased

b) Author to whom correspondence should be addressed; Electronic mail: young.kim@uni-konstanz.de
}

the properties of inorganic materials such as oxides converge to the bulk properties as a function of cluster size.

In this paper we demonstrate the existence of a class of materials, characterized by strong covalent or ionic bond, where very small clusters have the similar properties as their bulk, and thus can be used as a prototype system to understand some of the complex issues in surface science at the atomic scale. We concentrate on transition metal oxides, in particular tungsten oxide, $\mathrm{WO}_{3}$ in the present study. These materials are important for technological applications such as substrates for film growth, catalysis, and electrochromic devices. To shed light on variations of properties of oxide clusters as a function of cluster size, $\left(\mathrm{WO}_{3}\right)_{n}$ with $n=1-4$ are studied theoretically as well as experimentally. In contrast to the generally accepted view for the metallic clusters that more than 50-60 atoms are required for the onset of the bulk structures, geometric and electronic structures of $\left(\mathrm{WO}_{3}\right)_{4}$ cluster turned out to be nearly identical to that of the $\mathrm{WO}_{3}$ bulk crystal, indicating that the electronic properties of oxide clusters can converge to the bulk properties more rapidly than the pure metal clusters.

We show that the interatomic bond lengths, atomic arrangements, relative trends in stabilities, electronic structure, and energy gap between the highest occupied and lowest unoccupied molecular orbitals (this corresponds to the indirect band gap in bulk semiconductors) of $\left(\mathrm{WO}_{3}\right)_{4}$ cluster are nearly same as those in bulk tungsten oxide. In addition, a systematic study of $\mathrm{W}_{2} \mathrm{O}_{m}(m \leqslant 6)$ clusters shows that the $\mathrm{W}-\mathrm{W}$ bond is broken when the oxygen content reaches the bulk composition, namely, at $m=6$. The adiabatic electron affinities (AEA) and vertical detachment energies (VDE) of $\left(\mathrm{WO}_{3}\right)_{n}(n=1-4)$ clusters are nearly the same indicating that the bulk bonding characteristics are developed even in the smallest cluster having the bulk composition. These re- 
sults are based upon a combined theoretical and experimental study. The theoretical calculations are carried out using molecular orbital theory and the density functional formalism while the experimental studies involve mass ion intensities and photoelectron spectroscopy.

We begin with a summary of the structure and properties of bulk $\mathrm{WO}_{3}$. It is a semiconductor ${ }^{11}$ with an indirect band gap of $2.62 \mathrm{eV}$ and direct band gap of $3.5 \mathrm{eV}$. Here the six valence electrons of $\mathrm{W}$ originating from its $5 d^{5} 6 s^{1}$ configuration bond covalently with divalent oxygen. The crystalline structure of $\mathrm{WO}_{3}$ is complex: The basic building block is that of a simple cubic perovskitelike unit with the tungsten atom occupying the center of an octahedron containing corner and edge sharing oxygen atoms. As a function of temperature and pressure tungsten trioxide crystals exhibit at least five different phases (tetragonal $\rightarrow$ orthorhombic $\rightarrow$ monoclinic $\rightarrow$ triclinic $\rightarrow$ monoclinic). However, it is commonly accepted that $\mathrm{WO}_{3}$ has a monoclinic lattice at room temperature. As the tungsten oxide crystal is vaporized, ${ }^{12}$ it ejects $\mathrm{W}_{3} \mathrm{O}_{9}$ clusters indicating that these may be among the most stable structures having molecular architecture. For $\mathrm{WO}_{3}$ clusters on surfaces, three-membered rings of these octahedral units are observed. The three $\mathrm{W}$ atoms are connected via oxygen atoms forming a regular triangle with $\mathrm{W}-\mathrm{O}-\mathrm{W}$ sides. The main question is; do the structural and electronic properties of small tungsten oxide clusters bear any resemblance to their bulk and if so what is the critical size?

Unfortunately, there are no experimental techniques at the current time that can unambiguously yield the atomic structure of clusters. However, this information can be obtained from first principles calculations based on molecular orbital theory. Using density functional formulation we have calculated the equilibrium geometries and electronic properties of $\left(\mathrm{WO}_{3}\right)_{n}, n=1-4$ clusters as well as those of $\mathrm{W}_{2} \mathrm{O}_{m}(m \leqslant 6)$ clusters. The accuracy of these structures are validated by comparing the calculated binding energies, AEA and VDE with experiments involving mass ion intensity distributions and photoelectron spectroscopy. We show that small tungsten oxide clusters indeed exhibit the same structural and electronic properties as its bulk. In Secs. II and III we outline our theoretical and experimental procedures, respectively. The calculated results are compared with experiment in Sec. IV and summarized in Sec. V.

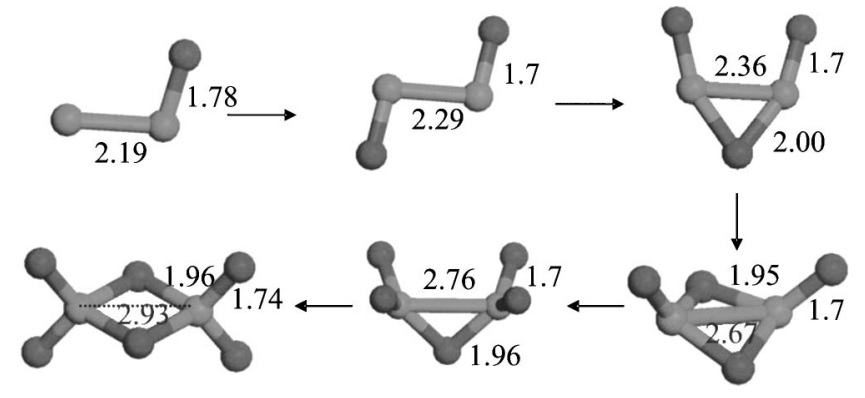

FIG. 1. Evolution of the equilibrium geometries of $\mathrm{W}_{2} \mathrm{O}_{m}(m=1-6)$ clusters. The $\mathrm{W}-\mathrm{W}$ and $\mathrm{W}-\mathrm{O}$ bond lengths in angstrom are as noted. The lighter shaded atom signifies $\mathrm{W}$ and darker atom represents oxygen.

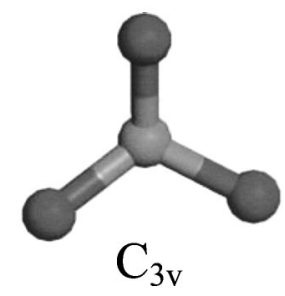

\section{$\mathrm{E}=0.0$ $3 \mathrm{D}$ structure}

(a)

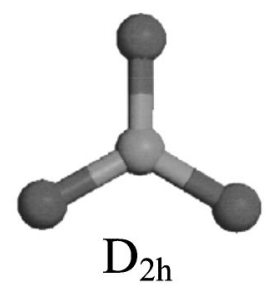

$$
\mathrm{E}=+0.95 \mathrm{eV}
$$$$
\text { 2D structure }
$$

(b)
FIG. 2. Structural isomers of anionic $\mathrm{WO}_{3}$ cluster. The relative energy $E$ is given.

\section{THEORY}

The theoretical calculations are carried out using the generalized gradient approximation (GGA) for exchangecorrelation potential in the density functional theory (DFT). The GGA form prescribed by Becke-Perdew-Wang (commonly referred to as BPW-91) was used. The atomic orbitals were represented by a Gaussian basis. We have used the 6-311G* basis set for oxygen and the Stuttgart relativistic effective core potential basis set for $\mathrm{W} .{ }^{13}$ The structures for both neutral and anionic clusters were optimized globally without any symmetry constraint and by starting from various initial configurations. The GAUSSIAN98 code was used in all these calculations. ${ }^{14}$

We first investigate the minimum amount of oxygen needed to cleave the $\mathrm{W}-\mathrm{W}$ bond in a tungsten oxide cluster. The simplest cluster in which to study the effect is $\mathrm{W}_{2} \mathrm{O}_{m}$, $m=1-6$ since there is only one $\mathrm{W}-\mathrm{W}$ bond and at $m=6$, the cluster attains the bulk stöichiometric composition. In Fig. 1 we show the equilibrium geometries including some representative bond lengths of $\mathrm{W}_{2} \mathrm{O}_{m}(m=1-6)$ clusters. With sequential addition of oxygen, two distinct types of $\mathrm{W}-\mathrm{O}$ bonds emerge: an internal bond where the $\mathrm{O}$ atom is attached to two $\mathrm{W}$ atoms and a terminal bond where the $\mathrm{O}$ atom is attached to a single $\mathrm{W}$ atom. In the bulk, these terminal bonds are absent. We note that the $\mathrm{W}-\mathrm{O}$ bond lengths remain essentially unchanged as a function of oxygen up-

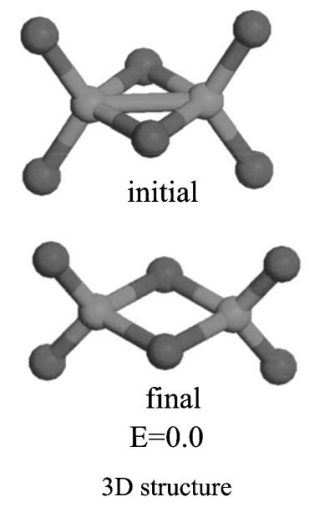

(a)

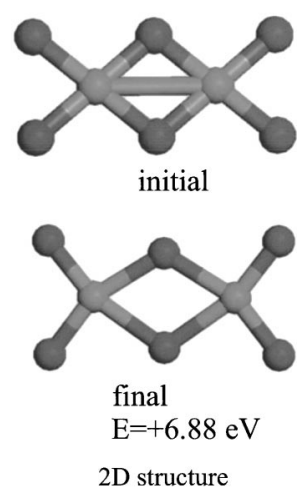

(b)
FIG. 3. Structural isomers of anionic $\left(\mathrm{WO}_{3}\right)_{2}$ cluster. 


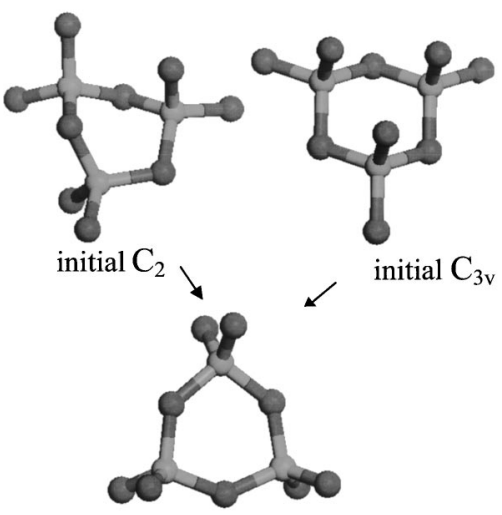

final $\mathrm{C}_{3 \mathrm{v}} \mathrm{E}=0$.

(a)

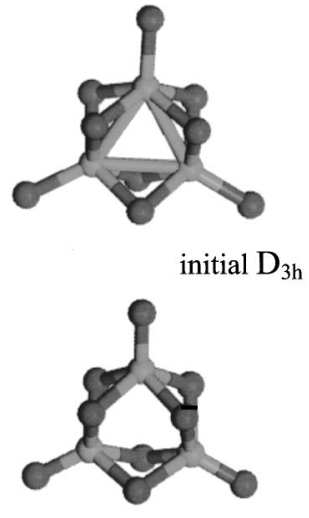

final $\mathrm{D}_{3 \mathrm{~h}} \mathrm{E}=+3.23 \mathrm{eV}$

(b)

FIG. 4. Structural isomers of anionic $\left(\mathrm{WO}_{3}\right)_{3}$ cluster.

take. The $\mathrm{W}-\mathrm{W}$ bond length begins to increase with oxygen content and breaks when the oxygen concentration reaches the bulk limit, namely, $\mathrm{W}_{2} \mathrm{O}_{6}$.

Next we discuss the geometries of the ground state and higher energy isomers of anion $\left(\mathrm{WO}_{3}\right)_{n}$ clusters in Figs. $2-5$. For clusters with $n=2,3,4$ we have given the initial starting geometries as well as final optimized geometries. In Fig. 2 we show the two isomers of $\mathrm{WO}_{3}$ cluster. The threedimensional structure $\left(C_{3 v}\right)$ is more stable than the twodimensional structure $\left(D_{2 h}\right)$ by $0.95 \mathrm{eV}$. This is also true for $n=2$ (Fig. 3) where the three-dimensional structure is more stable than the two-dimensional structure, and the latter lies $6.88 \mathrm{eV}$ higher in energy than the former. For $n=3$, we considered three initial starting geometries. The two initial geometries with $C_{2}$ and $C_{3 v}$ symmetries converged to the same structure upon relaxation [Fig. 4(a)]. When starting from the $D_{3 h}$ geometry, the resulting optimized structure in Fig. 4(b) lies $3.23 \mathrm{eV}$ higher in energy than the structure in Fig. 4(a). For $n=4$, we found that eight different initial structures con-

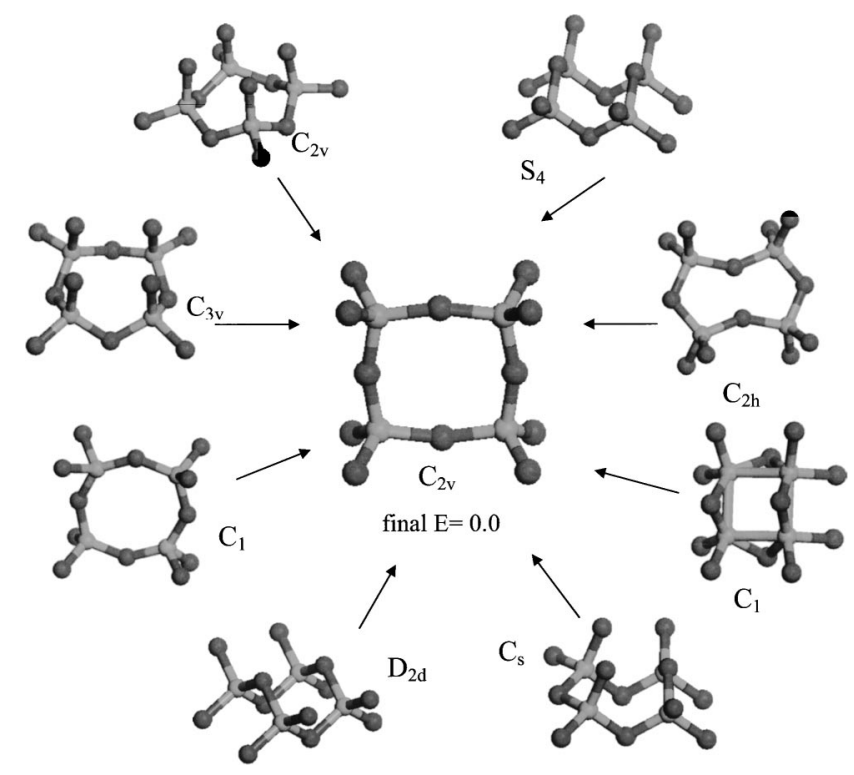

FIG. 5. Initial and final optimized structures of anionic $\left(\mathrm{WO}_{3}\right)_{4}$ cluster.
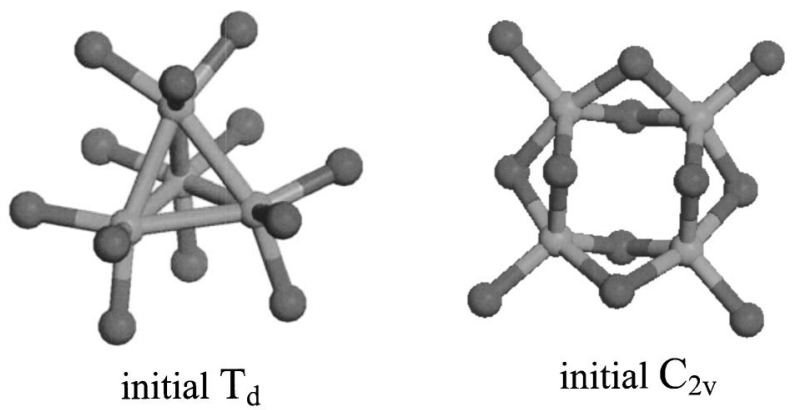

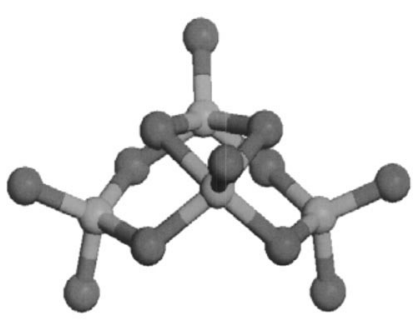

final $\mathrm{C}_{2 \mathrm{v}} \mathrm{E}=+0.723 \mathrm{eV}$

(a)

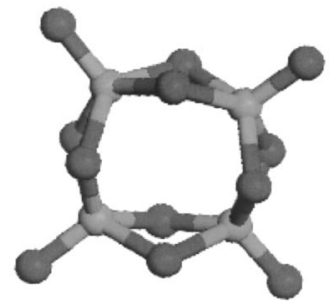

final $\mathrm{C}_{2} \quad \mathrm{E}=+1.825 \mathrm{eV}$

(b)
FIG. 6. Geometries of higher energy isomers of anionic $\left(\mathrm{WO}_{3}\right)_{4}$ cluster. The relative energy $E$ is respective to the ground geometry in Fig. 5 .

verged to the same one (Fig. 5) with $C_{2 v}$ symmetry. We also found two other higher energy isomers of $\left(\mathrm{WO}_{3}\right)_{4}$ cluster (see Fig. 6). These lie $0.72 \mathrm{eV}$ and $1.83 \mathrm{eV}$ above the ground state structure in Fig. 5. However, there are some common features shared by the ground state structures studied: (1) The $\mathrm{W}-\mathrm{W}$ bond is always broken by oxidation. (2) As will be discussed later, the stable structures have large HOMOLUMO (highest occupied molecular orbital-lowest unoccupied molecular orbital) gaps.

The ground state geometries of the anions of $\left(\mathrm{WO}_{3}\right)_{4}$ clusters $(n=1-4)$ are compared with the corresponding neutrals in Figs. 7-10. Note that the geometries of anion

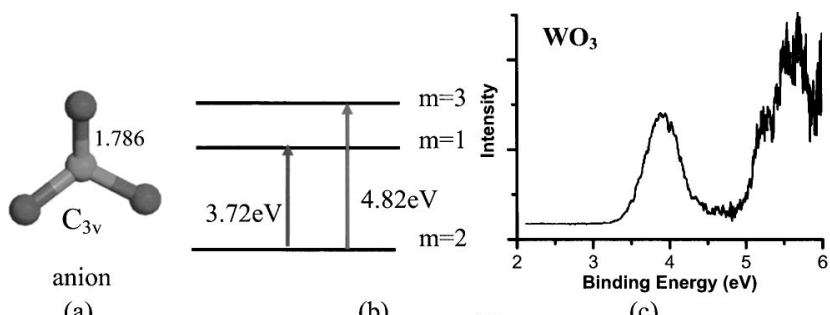

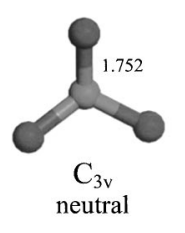

(d)

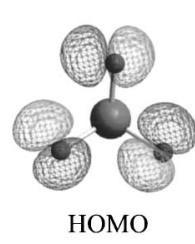

(e)

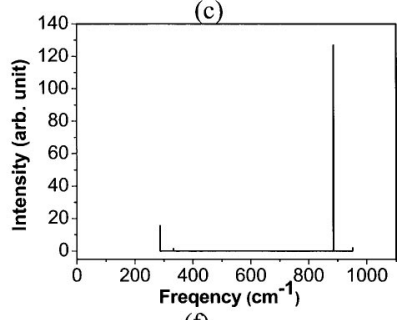

(f)
FIG. 7. Theoretical and experimental data on $\mathrm{WO}_{3}$ cluster. (a) Ground state geometry of the anion. (b) The calculated vertical detachment energies from the anion ground state with spin multiplicity $M$ to the neutral with spin multiplicity $M^{\prime}=M \pm 1$. (c) Photodetachment spectra. (d) Ground state geometry of the neutral. (e) HOMO. (f) Vibrational spectrum. 


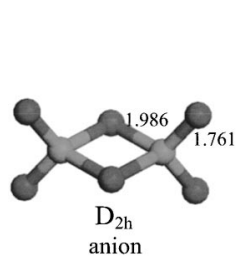

(a)

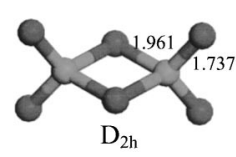

neutral

(d)

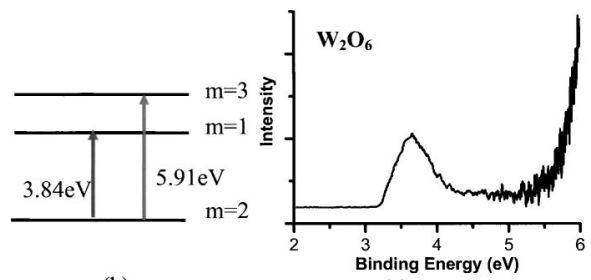

(b)

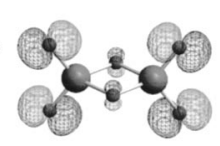

HOMO

(e)

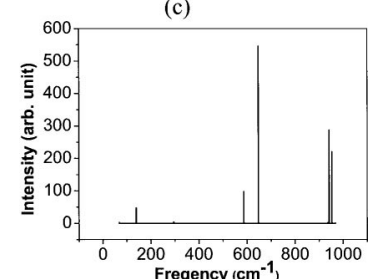

(f)
FIG. 8. Theoretical and experimental data on $\left(\mathrm{WO}_{3}\right)_{2}$ cluster. See Fig. 7 for rest of the legend.

[Figs. 7(a)-10(a)] and neutral [Figs. 7(d)-10(d)] clusters are nearly the same with the anions having marginally enlarged bonds. There are no $\mathrm{W}-\mathrm{W}$ bonds in any of these clusters. The internal $\mathrm{W}-\mathrm{O}$ bond is slightly larger than the terminal $\mathrm{W}-\mathrm{O}$ bond. The frequency calculations for the neutral structures given in Figs. 7(f)-10(f) show that all the frequencies are positive. Thus the structures are dynamically stable. The HOMOs of all the clusters given in Figs. 7(e)-10(e) are contributed by $\mathrm{O}-2 p$ orbitals. The structure of $\mathrm{W}_{3} \mathrm{O}_{9}$ cluster [Fig. 9(d)], characterized by a ring with three $\mathrm{W}$ atoms and three $\mathrm{O}$ atoms forming regular triangles, is nearly identical to that observed on surfaces.

To further demonstrate how closely these clusters resemble the bulk structure, we compare the geometry of $\mathrm{W}_{4} \mathrm{O}_{12}$ cluster with that cut out from the monoclinic structure of bulk tungsten oxide in Fig. 11. The internal $\mathrm{W}-\mathrm{O}$ bond length in the $\mathrm{W}_{4} \mathrm{O}_{12}$ cluster is $1.92 \AA$, which is very close to the bulk value of $1.89 \AA$. Since in the free cluster the terminal $\mathrm{W}-\mathrm{O}$ bonds are different from the internal bond due to lack of proper coordination, and in bulk $\mathrm{WO}_{3}$ the $\mathrm{W}-\mathrm{O}$ bonds form zigzag chains with varying bond distances, we

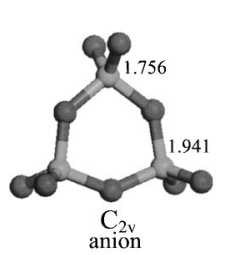

(a)

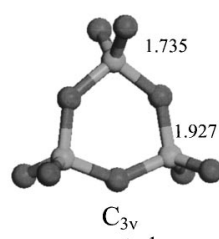

(d)

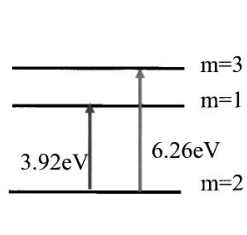

(b)

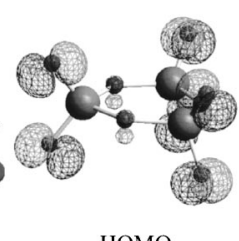

(e)

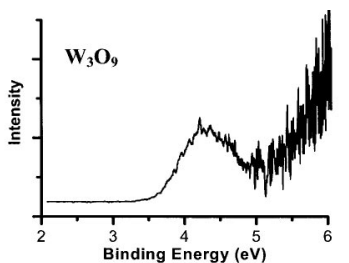

(c)

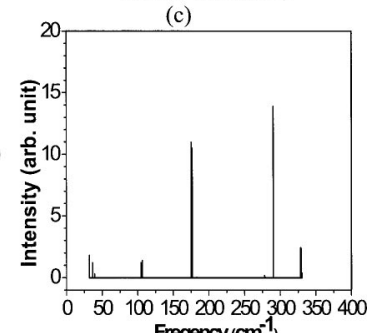

(f)
FIG. 9. Theoretical and experimental data on $\left(\mathrm{WO}_{3}\right)_{3}$ cluster. See Fig. 7 for rest of the legend.

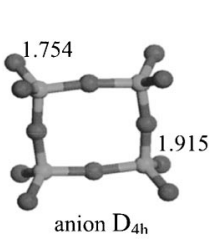

(a)

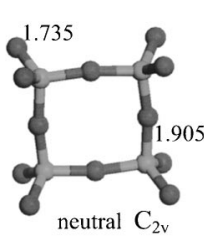

(d)

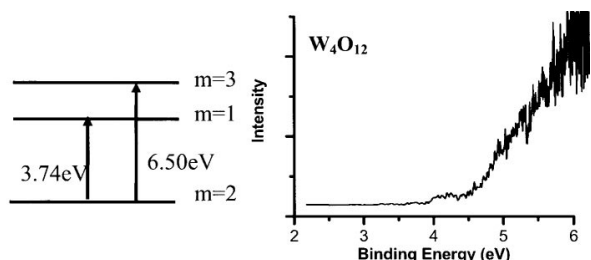

(b)

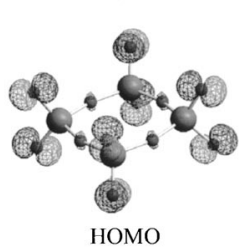

(e)

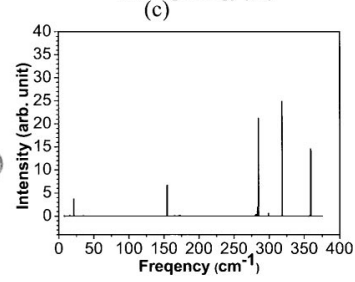

(f)
FIG. 10. Theoretical and experimental data on $\left(\mathrm{WO}_{3}\right)_{4}$ cluster. See Fig. 7 for rest of the legend.

have calculated the average $\mathrm{W}-\mathrm{O}$ bond length. For a free cluster, it is $1.83 \AA$ while for the monoclinic crystal, the same average yields $1.80 \AA$. The nearest $\mathrm{W}-\mathrm{W}$ interatomic distance in the $\mathrm{W}_{4} \mathrm{O}_{12}$ cluster is $3.80 \AA$ while it is $3.7 \AA$ in bulk $\mathrm{WO}_{3}$. Similarly, the two $\mathrm{O}-\mathrm{W}-\mathrm{O}$ bond angles in $\mathrm{W}_{4} \mathrm{O}_{12}$ cluster agree very well with those in the crystalline phase. These comparisons clearly demonstrate that the geometry of the $\mathrm{W}_{4} \mathrm{O}_{12}$ cluster is as if it is a fragment of the bulk.

\section{EXPERIMENTS}

Unfortunately, no experimental technique exists that can verify the structural parameters predicted by theory. However, the accuracy of the theoretically determined structures can be established by comparing the calculated AEA, VDE, HOMO-LUMO gap, and relative stabilities of clusters with experiments involving photoelectron spectra and mass ion intensity distribution. Experimental setup used to obtain photoelectron spectra of tungsten oxide clusters are described in detail elsewhere. ${ }^{15} \mathrm{~W}_{n}$ anions are produced with a PACIS (pulsed arc cluster ions source) by vaporizing bulk $\mathrm{W}$ in the presence of oxygen. The anions are mass selected using a reflectron time-of-flight spectrometer (mass resolution $m / \Delta m=400)$. A mass-selected bunch is irradiated by a UV laser pulse $(h v=4.66 \mathrm{eV})$ and the kinetic energies of the detached electrons are measured using a "magnetic bottle"type time-of-flight electron spectrometer. The energy resolution is about $2 \%$ corresponding to $20 \mathrm{meV}$ at a kinetic energy of $1 \mathrm{eV}$. It is important to mention that binding energies of peaks in photoelectron spectra provide information on tran-
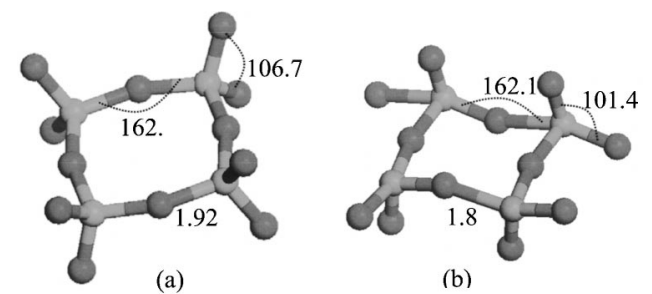

(b)

FIG. 11. Comparison between the equilibrium geometry of (a) $\mathrm{W}_{4} \mathrm{O}_{12}$ cluster and (b) the corresponding fragment taken from the monoclinic phase of bulk $\mathrm{WO}_{3}$. 


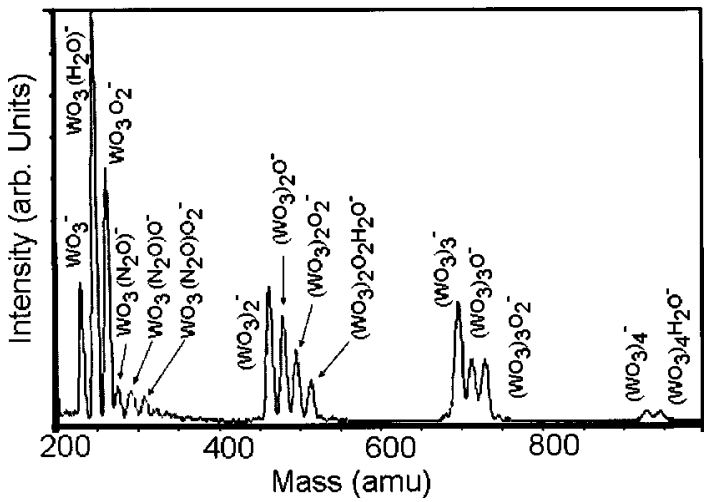

FIG. 12. Experimental mass spectra.

sition energies from the ground state of an anionic cluster to the ground and excited states of the neutral counterpart with the same geometry as that of the anion ground state. This information is also accessible in our theoretical calculations, allowing direct comparison of the experimental and theoretical results. One can first compute total energy and spin multiplicities $M=2 S+1$ of the anion in its ground state. Then the total energies of the neutral at the anion geometry but corresponding to spin multiplicities $M^{\prime}=M \pm 1$ can be calculated, yielding two transition energies corresponding to the ground and the first excited state of the neutral with the same geometry as the anion ground state. The HOMO-LUMO gap thus calculated as the energy difference between the two spin mulitiplets of the neutral cluster can be compared with experiment. Note that the HOMO-LUMO gaps calculated from the difference between the energy levels of the HOMO and LUMO orbitals in the DFT formalisms are not accurate as the single particle levels have no formal meaning. However, computing the gaps from the total energy difference as described above do not suffer from this shortcoming of the DFT.

The reliability of the theoretical approach used in the present wok can also be tested by comparing the calculated relative stabilities of the anionic clusters discussed above with experiments measuring the relative mass ion intensities. These experiments were conducted by producing cluster anions from heated filaments of the metal over which trace quantities of oxygen and water vapor were passed in a steady stream of helium maintained at a pressure of about 0.5 torr. The clusters were subjected to thousands of collisions, and hence are well thermalized at room temperature. Hence, under these quasisteady state conditions, the relative mass abundances are expected to be governed by the relative stabilities of the clusters of proximate size and composition. The distributions are plotted in Fig. 12. Note that the peak height of $\mathrm{W}_{2} \mathrm{O}_{6}^{-}$is much larger than that of $\mathrm{W}_{4} \mathrm{O}_{12}^{-}$, implying that $\mathrm{W}_{2} \mathrm{O}_{6}^{-}$is more stable than $\mathrm{W}_{4} \mathrm{O}_{12}^{-}$. As will be shown in the following section, this is in good accord with the calculated trends in bond energies.

\section{COMPARISON BETWEEN THEORETICAL AND EXPERIMENTAL RESULTS}

We first compare our calculated AEA (difference between the ground state energies of the anion and neutral), the VDE (difference between the energies of the anion and the neutral at the anion geometry), and energy gap (difference in energy between the two lowest spin multiplets of the neutral at the anion geometry) with experiment. The photodetachment spectra of $\left(\mathrm{WO}_{3}\right)_{n}(n=1-4)$ clusters are given in Figs. $7(\mathrm{c})-10(\mathrm{c})$. The UPS (一 ultraviolet photoemission spectroscopy) spectra of $\mathrm{WO}_{3}$ in Fig. 7(c) exhibit two features at binding energies of 3.8 and $5.2 \mathrm{eV}$, and these values are consistent with the calculated ones in Fig. 7(b) for the transitions from the anion ground state to the ground and first excited states of the neutral counterpart. For the remaining $\left(\mathrm{WO}_{3}\right)_{n}$ clusters, the calculated transition energies agree well with the experimental data [see Figs. 8(b) and 8(c)Figs. 10(b) and 10(c)]. To get a better perspective on the quantitative accuracy of the calculations, we show in Table I a comparison of the calculated and experimental AEA and VDE. The calculated energies agree with experiment to within $0.3 \mathrm{eV}$. This agreement is considered to be very good particularly when one realizes that in our calculations the treatment of $\mathrm{W}$ is not fully relativistic, the exchangecorrelation functional is not exact, and there are always inherent limitations (such as choice of basis sets) in a numerical procedure. The uncertainties in experimental values are $\pm 0.1 \mathrm{eV}$.

The mass-resolution of our experimental setup used for the UPS measurements is not sufficiently high to exclude the possibility for tungsten oxide clusters being protonated. However, previous studies on oxygen adsorption over various metal clusters performed using the same experimental setup show that concentrations of impurities such as hydro-

TABLE I. Calculated W-W and W-O nearest neighbor distance, adiabatic electron affinity (AEA), vertical detachment energy (VDE), and energy gap of $\left(\mathrm{WO}_{3}\right)_{n}(n=1-4)$ clusters compared with those available in the bulk monoclinic phase. Also listed for comparison are our experimental data.

\begin{tabular}{|c|c|c|c|c|c|c|c|c|c|}
\hline \multirow[b]{3}{*}{ Cluster } & \multirow[b]{3}{*}{$R_{\mathrm{W}-\mathrm{W}}(\AA)$} & \multirow[b]{3}{*}{$R_{\mathrm{W}-\mathrm{O}}(\AA)$} & \multirow{2}{*}{\multicolumn{2}{|c|}{ AEA $(\mathrm{eV})$}} & \multirow{2}{*}{\multicolumn{2}{|c|}{ VDE (eV) }} & \multicolumn{3}{|c|}{ Energy gap (eV) } \\
\hline & & & & & & & \multirow{2}{*}{$\begin{array}{c}\text { From } \\
\text { transition } \\
\text { energies }\end{array}$} & \multirow{2}{*}{$\begin{array}{l}\text { From } \\
\text { energy } \\
\text { levels }\end{array}$} & \multirow[b]{2}{*}{ Expt. } \\
\hline & & & Theo. & Expt. & Theo. & Expt. & & & \\
\hline $\mathrm{WO}_{3}$ & & & 3.31 & 3.48 & 3.72 & 3.83 & 1.10 & 1.68 & 1.5 \\
\hline$\left(\mathrm{WO}_{3}\right)_{2}$ & 2.92 & 1.96 & 3.74 & 3.40 & 3.84 & 3.65 & 2.07 & 2.17 & 2.5 \\
\hline$\left(\mathrm{WO}_{3}\right)_{3}$ & 3.57 & 1.93 & 3.29 & 3.45 & 3.92 & 4.12 & 2.34 & 3.45 & $>1.5$ \\
\hline$\left(\mathrm{WO}_{3}\right)_{4}$ & 3.80 & 1.92 & 3.42 & 3.69 & 3.74 & 4.00 & 2.76 & 3.46 & $>1.2$ \\
\hline Bulk & 3.7 & 1.89 & & & & & & & $2.62-3.5$ \\
\hline
\end{tabular}


gen and water are negligibly small in the cluster source, and therefore protonation of the clusters in our experiments is unlikely. ${ }^{16,17}$

To investigate the extent to which clusters yield the electronic properties of the bulk, we compare in Table I the calculated energy gaps with experiment. We obtain this gap first from the difference in the two calculated transition energies as described before. This can also be obtained directly from the single particle energy levels of the cluster as the difference between the energies of LUMO and HOMO. Note that in DFT these energy levels have no fundamental meaning. Yet, we have given these values in the last column of Table I not only to compare it with those obtained from total energy calculations, but also from experiment. We note that both calculated gaps increase with cluster size. For $\mathrm{WO}_{3}$ and $\left(\mathrm{WO}_{3}\right)_{2}$ clusters, the energy gaps calculated using two different procedures agree well with each other and with experiment. For $\left(\mathrm{WO}_{3}\right)_{3}$ and $\left(\mathrm{WO}_{3}\right)_{4}$ clusters, the experiments only predict a lower limit and both calculated gaps are consistent with this observation. It is interesting to note that for $\left(\mathrm{WO}_{3}\right)_{4}$ cluster the gap calculated from transition energies agree with the bulk indirect gap while that calculated from the energy levels agree with the direct gap.

We have studied the relative stabilities of the neutral and anionic clusters by calculating their total energies and computing from it the energy gained in adding a $\mathrm{WO}_{3}$ unit to a preexisting cluster having the same stöichiometric composition, namely,

$$
\begin{aligned}
& \Delta E=-E\left(\mathrm{WO}_{3}\right)_{n}+E\left(\mathrm{WO}_{3}\right)_{n-1}+E(\mathrm{WO})_{3}, \\
& \Delta E^{-}=-E\left(\mathrm{WO}_{3}\right)_{n}^{-}+E\left(\mathrm{WO}_{3}\right)_{n-1}+E\left(\mathrm{WO}_{3}\right)^{-} .
\end{aligned}
$$

The relative energies $\Delta E$ of the neutral clusters are $4.52 \mathrm{eV}$, $4.61 \mathrm{eV}$, and $3.82 \mathrm{eV}$, respectively, for $\mathrm{W}_{2} \mathrm{O}_{6}, \mathrm{~W}_{3} \mathrm{O}_{9}$, and $\mathrm{W}_{4} \mathrm{O}_{12}$. This indicates that formation of neutral $\mathrm{W}_{3} \mathrm{O}_{9}$ cluster is energetically preferred over that of other clusters. Thus, one would expect that in laser ablation of bulk tungsten oxide, the $\mathrm{W}_{3} \mathrm{O}_{9}$ units would be ejected. This is, indeed, what has been observed experimentally. ${ }^{12}$ The relative energy gains $\Delta E^{-}$, for the anionic $\left(\mathrm{WO}_{3}\right)_{n}^{-}$clusters are $4.96 \mathrm{eV}$, $4.59 \mathrm{eV}$, and $3.94 \mathrm{eV}$ for $n=2,3$, and 4, respectively. This implies that among anionic clusters, $\mathrm{W}_{2} \mathrm{O}_{6}^{-}$should be energetically more favored than $\mathrm{W}_{4} \mathrm{O}_{12}^{-}$. This is also consistent with the mass ion intensity distribution in Fig. 12.

\section{CONCLUSIONS}

In summary, we find that (1) the $\mathrm{W}-\mathrm{W}$ bonds are broken when the oxygen content of the cluster reaches the bulk limit, namely, in $\left(\mathrm{WO}_{3}\right)_{n}(n=2,3,4)$ clusters. (2) Among the neutral clusters studied, $\mathrm{W}_{3} \mathrm{O}_{9}$ is the most stable one while $\mathrm{W}_{2} \mathrm{O}_{6}^{-}$is more stable than $\mathrm{W}_{2} \mathrm{O}_{9}^{-}$and $\mathrm{W}_{4} \mathrm{O}_{12}^{-}$. (3) The $\mathrm{W}-\mathrm{O}$ and $\mathrm{W}-\mathrm{W}$ bond lengths and $\mathrm{O}-\mathrm{W}-\mathrm{O}$ bond angles of $\mathrm{W}_{4} \mathrm{O}_{12}$ cluster are nearly the same as those in the bulk. (4) The
HOMO-LUMO gap calculated using the energy difference between the HOMO and LUMO energy levels agrees well with those obtained from the total energy difference. The "band gap" of $\mathrm{W}_{4} \mathrm{O}_{12}$ cluster is very similar to that in the bulk as well. Thus $\mathrm{W}_{4} \mathrm{O}_{12}$ is an embryonic form of bulk tungsten oxide. We should point out that previous mass spectroscopy studies of alkali halides have demonstrated that clusters with conspicuously high intensities (magic numbers) can be identified with small fragments of its bulk. Thus, the geometries of these magic clusters may resemble those of their bulk. However, no studies of their electronic properties, as reported here, have been carried out. They will be useful.

This demonstration that a small cluster of a class of materials with strong covalent or ionic bond, behaves same as their bulk has important implications. It is well known that an atomic level understanding of catalysis in bulk materials is difficult due to presence of defects and problems with proper characterization. In atomic clusters this does not arise as mass spectroscopy can identify the precise atomic composition of a cluster and photoelectron spectroscopy can yield accurate energies. Thus once one can identify the size of a cluster that has all the attributes of its bulk, its reaction kinetics can be studied with conventional cluster techniques. It is hoped that such a study can illustrate the reactivity of clusters that has relevance to bulk systems. It is important to point out that for such an understanding, the synergy between theory and experiment is necessary.

${ }^{1}$ H. W. Kroto, J. R. Heath, S. C. O'Brien et al., Nature (London) 318, 162 (1985).

${ }^{2}$ K. M. Ho, A. A. Shvartsburg, B.-C. Pan et al., Nature (London) 392, 582 (1998).

${ }^{3}$ R. F. Service, Science 271, 920 (1996).

${ }^{4}$ J. Uppenbrink, Science 292, 2401 (2001).

${ }^{5}$ B. C. Guo, K. P. Kerns, and A. W. Castleman, Jr., Science 255, 1411 (1992).

${ }^{6}$ B. V. Reddy, S. N. Khanna, and P. Jena, Science 258, 1640 (1992).

${ }^{7}$ T. P. Martin, T. Bergmann, H. Göhlich, and T. Lange, Chem. Phys. Lett. 172, 209 (1990).

${ }^{8}$ K. Raghavachari and C. M. Rohlfing, J. Chem. Phys. 94, 3670 (1991).

${ }^{9}$ J. Müller, B. Liu, A. A. Shvartsburg, S. Ogut, J. R. Chelikowsky, K. W. M. Siu, K.-M. Ho, and G. Ganteför, Phys. Rev. Lett. 85, 1666 (2000).

${ }^{10}$ K. J. Taylor, C. L. Pettiette, O. Cheshnovsky, and R. E. Smalley, J. Chem. Phys. 96, 3319 (1992).

${ }^{11}$ F. P. Koffyberg, K. Dwight, and A. Wold, Solid State Commun. 30, 433 (1979).

${ }^{12}$ J. Berkowitz, W. A. Chupka, and M. G. Inghram, J. Chem. Phys. 27, 85 (1957).

${ }^{13}$ M. Dolg, H. Stoll, H. Preuss, and R. M. Pitzer, J. Phys. Chem. 97, 5852 (1993).

${ }^{14}$ M. J. Frish, G. W. Trucks, H. B. Schlegel et al., GAussian 98, Gaussian, Inc., Pittsburgh, PA, 1998.

${ }^{15}$ H. Handschuh, G. Ganteför, and W. Eberhardt, Rev. Sci. Instrum. 66, 3838 (1995).

${ }^{16}$ D. Stolcic, M. Fischer, G. Ganteför, Y. D. Kim, Q. Sun, and P. Jena, J. Am. Chem. Soc. 125, 2848 (2003)

${ }^{17}$ Y. D. Kim, M. Fischer, and G. Ganteför, Chem. Phys. Lett. 377, 170 (2003). 